\section{INTRATUMORAL ADMINISTRATION AND LOCAL RETENTION OF IL-2/IL-12 FUSION PROTEINS DRIVE A POTENT SYSTEMIC ANTI-TUMOR IMMUNE RESPONSE}

${ }^{1}$ Mehta Naveen*, 'Bochong Li, ${ }^{2}$ Dane Wittrup, ${ }^{1}$ Patrick Baeuerle, ${ }^{1}$ Jennifer Michaelson. ${ }^{1}$ Cullinan Amber Corp., Cambridge, MA, USA; ${ }^{2}$ MIT, Cambridge, MA, USA

Background IL-2 and IL-12 synergistically trigger the stimulation and proliferation of $\mathrm{T}$ and $\mathrm{NK}$ cells to mediate antitumor immunity. Although aldesleukin, a high-dose IL-2 intravenous (IV) infusion regimen, has been approved for the treatment of melanoma and renal cell carcinoma, adoption has been hindered by frequent grade 3 and 4 severe adverse events. No IL-12 therapy has been approved yet due to toxicity. Cullinan Amber is developing a fusion protein that uniquely combines in one polypeptide both IL-2 and IL-12 with a collagen-binding domain to reduce toxicity and increase efficacy following intra-tumoral (IT) administration via retention in the tumor microenvironment.

Methods Proteins were expressed in HEK293 cells. Collagen binding was measured by ELISA. IL-2 and IL-12 bioactivity was evaluated by CTLL-2 proliferation and HEK-Blue IL-12 reporter cells. In vivo studies were conducted in B16F10, MC38, and CT26 syngeneic tumor models. Systemic Amber construct concentrations were determined by ELISA.

Results "Amber" constructs, comprised of IL-2, IL-12, and a collagen-binding domain, were produced and confirmed to retain bioactivity. B16F10 tumor-bearing mice injected with Amber IT had systemic Amber levels $<5 \%$ as compared to mice administered the same dose IV. When IL-2/IL-12 fusion proteins lacking a collagen-binding domain were injected IT in B16F10-bearing mice, $60 \%$ of mice needed to be euthanized due to severe body weight loss, while Amber-treated mice did not lose body weight. In the checkpoint-refractory B16F10 and MC38 models, Amber demonstrated 95\% tumor growth inhibition (figure 1a) and 100\% CRs (figure 1b), respectively. $90 \%$ of the mice cured of their primary MC38 tumors were protected from re-challenge (figure 1b). Notably, 70\% CRs were observed in the MC38 model even after a single-dose treatment of Amber. Similar data was obtained in the CT26 model. Amber treatment of mice bearing large $500 \mathrm{~mm} 3$ MC38 tumors resulted in dramatic tumor shrinkage (figure 1c). In mice bearing two MC38 tumors, only one of which was treated IT, $100 \%$ of treated tumors and $90 \%$ of distal untreated tumors were eliminated when Amber was combined with an anti-PD1 antibody (figure 1d), demonstrating a robust abscopal response.
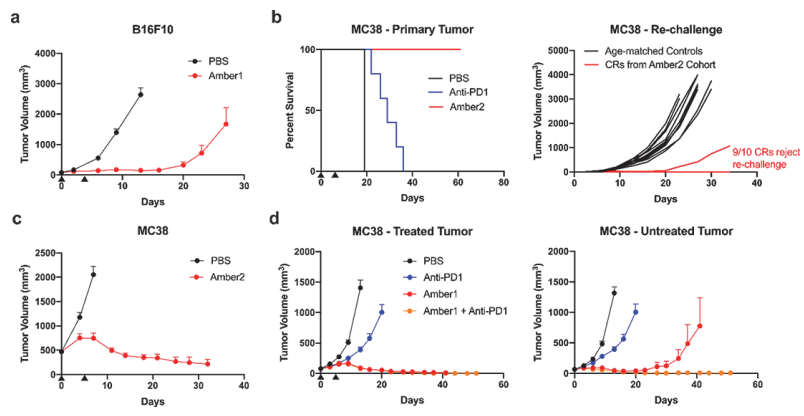

Abstract 876 Figure 1 Efficacy of amber constructs in syngeneic tumor models
Conclusions The use of collagen-binding domains for tumor retention enables the safe and effective delivery of IL-2 and IL-12 in a single multifunctional molecule. Taken together, the preclinical data suggests that Amber constructs may show robust single-agent activity in clinical trials against checkpointrefractory tumors with minimal toxicity, as well as the potential to significantly deepen anti-tumor responses in combination with checkpoint inhibitor therapy.

http://dx.doi.org/10.1136/jitc-2021-SITC2021.876 